\title{
Update on HE vs UHE Collimation for Focal Total-activity Quantification in I-131 SPECT Using 3D OSEM
}

\author{
Kenneth F. Koral, Member IEEE, Anastasia Yendiki, Victor Lin, Yuni K. Dewaraja, and Jeffrey A. \\ Fessler, Member IEEE
}

\begin{abstract}
We calibrated a scintillation camera for the counts-toactivity conversion factor, $\mathrm{CF}$, by measuring a phantom consisting of a sphere containing a known 131-I activity placed within an elliptical cylinder. Within a $3 D$ OSEM reconstruction algorithm, we employed a depth-dependent detector-response model based on smooth fits to the point-source-response function. Using the ultrahigh-energy (UHE) collimator and 100 iterations, the recovery coefficient, $R C$, appeared to be 1 for any sphere volume down to 20 cm3. The CF changed only a small amount as the backgroundover-target activity concentration ratio, $b$, increased for both $U H E$ and high-energy (HE) collimation. Tests of activity quantification were carried out with an anthropomorphic phantom simulating a $100 \mathrm{~cm} 3$ spherical tumor centrally located inferior to the lungs. With $3 D$ OSEM reconstruction, using the global-average $C F$ and no RC-based correction, mean bias in the simulated-tumor activity estimate over 20 realizations was $-7.4 \%$ with $\mathrm{UHE}$ collimation, and $-9.4 \%$ with $H E$ collimation. For comparison, with $1 D$ SAGE reconstruction, using the $C F$ corresponding to the experimental estimate of b and RC-based correction, the mean bias was worse, $10.7 \%$ for UHE collimation, but better, $-4.3 \%$, for $\mathrm{HE}$ collimation.
\end{abstract}

\section{INTRODUCTION}

Our interest is in SPECT activity quantification of focal I-131 uptake within a volume of interest ( VoI) to estimate total activity in tumors during radiopharmaceutical therapy. We employ a phantom-based calibration of the camera using a

Manuscript received Oct 28, 2003. This research was supported by grants RO1 CA87955 and CA80927 awarded by the National Cancer Institute, U.S. Department of Health and Human Services.

K. F. Koral is with the Department of Radiology, The University of Michigan, Ann Arbor, MI 48109-0552 USA (telephone: 734-764-5103, email:kenkoral@umich.edu).

A. Yendiki is with the Department of Electrical and Electronic Engineering, The University of Michigan, (e-mail: nastazia@eecs.umich.edu).

V. Lin was with the Department of Radiology, The University of Michigan, (e-mail: VQiang@Plastipak.com).

Y. K. Dewaraja is with the Department of Radiology, The University of Michigan, (e-mail: yuni@umich.edu).

J. A. Fessler is with the Department of Electrical and Electronic Engineering, The University of Michigan, (e-mail: fessler@eecs.umich.edu). sphere with known activity in an elliptical phantom. The total counts within a spherical $\mathrm{VoI}$ in the reconstructed image yields the counts to-activity-conversion factor, $\mathrm{CF}$, for a particular camera-collimator combination. The spherical $\mathrm{VoI}$ is drawn on the CT image and located in the SPECT image by a markerbased CT-SPECT registration. We had used a regularized 1D Space-Alternating Generalized EM (SAGE) algorithm with attenuation correction but without detector-response modeling [1], [2] for our previous patient imaging [3]. Since that work, we have been investigating the inherently-unregularized Ordered-Subset Expectation Maximization (OSEM) algorithm [4] with depth-dependent detector-response modeling [5] to improve image resolution. Those investigations have involved both an ultra-high-energy (UHE) collimator that was originally designed for gamma-camera positron imaging and the highenergy (HE) collimator that is usually used for I-131 imaging. The most significant I-131 gamma-ray emission is at $364 \mathrm{keV}$ $(82 \%)$. However, emissions at $637 \mathrm{keV}(7.2 \%)$ and $723 \mathrm{keV}$ (1.8\%) also exist and can penetrate the septa of the usual HE parallel-hole collimator. The UHE collimator has reduced septal penetration compared to the HE collimator [6].

We recently found that we needed to change our OSEM algorithm to handle edge effects in order to eliminate an artifact in patient reconstructions. The change also affected the phantom-based calibrations previously reported[7]-[8]. With the new version of the algorithm, the camera calibration results for the UHE collimator have been detailed in a separate manuscript submitted for publication [9]. In this paper, we summarize those results and present a comparison of the UHE calibration to that for the HE collimator. We also evaluate activity results from multiple realizations of a test phantom, with quantification based on OSEM for each collimator and compare those to quantifications based on SAGE for the same data.

\section{METHODS}

To calibrate the Prism 3000 triple-headed gamma camera, SPECT images of a $200 \mathrm{~cm}^{3}$, known-I-131-activity sphere situated off center along the long axis in an elliptical water 
phantom were acquired into a $64 \times 64$ matrix. The sphere contained $14.5 \mathrm{MBq}(391 \mu \mathrm{Ci})$ of I-131. Uniformbackground-activity level in the water was varied to produce four $\mathrm{b}$ values, ranging from 0 to 0.37 . Here $\mathrm{b}$ is defined as the ratio of the activity concentration in the water of the cylinder divided by that in the water of the sphere. For the UHE collimator, we used a 120-degree circular-orbit with 6 degrees between projections and combined the data to get 360-degree data for a reconstruction. Each phantom acquisition was carried out at five values of the radius of rotation, $\mathrm{R}(19,21$, $23,24.5$ and $26 \mathrm{~cm}$ ). For the HE collimator, we used a 360degree circular-orbit. In this summary, we preliminarily inferred the counts and conversion factor for the entire camera from the head 1 data alone (counts and CF from head 1 were simply multiplied by 3 ). A more accurate sum of the results from the 3 heads will be carried out later. Two R values (22 and $26 \mathrm{~cm}$ ) were investigated.

To model detector response for 3D OSEM reconstruction, we used experimental point-source measurements acquired for head 1 as a function of distance into a $512 \times 512$ matrix. For the UHE collimator, the average behavior of the point source response was modeled by a rotationally-symmetric Gaussian. Width and center location of the Gaussian were determined by non-linear least-squares fitting. For the $\mathrm{HE}$ collimator, a rotationally-symmetric single exponential was added to the Gaussian to model septal penetration. Due to the relative simplicity of the functions, and an assumption of shift invariance on a plane parallel to the detector, the detailed hole pattern was not taken into account.

Reconstruction was carried out with 1) SAGE using a shiftinvariant strip integral system model (1D SAGE); 2) an OSEM algorithm driven by a 3D matrix, each slice of which was associated with a depth (3D OSEM). The change to the $3 \mathrm{D}$ OSEM algorithm was that edges of the volume were handled by assuming zero values outside the volume in the transverse $(\mathrm{x}-\mathrm{y})$ directions and repetition of the appropriate end slice in the + and $-\mathrm{z}$-direction.

Monte-Carlo simulation [10]-[11] generated SPECT data for the UHE collimator to yield recovery coefficients for spherical volumes different than $200 \mathrm{~cm}^{3}$. A matrix size of $64 \times 64$, and three different values of uniform background were investigated, but radius of rotation was not varied. The pixelated VoI was determined from the known location and radius of the simulated spherical activity.

As a test of the accuracy of the quantification scheme based on 3D OSEM compared to that based on 1 D SAGE, a $100 \mathrm{~cm}^{3}$ sphere containing $28.9 \mathrm{MBq}(780 \mu \mathrm{Ci})$ of I-131 and centrally located in an anthropomorphic lung phantom was sequentially imaged 20 times with each collimator. A 120 deg rotation was used with both collimators. For each collimator, the $3 \mathrm{D}$ OSEM CF was the global average of the CF's measured for the different R,b values. No correction for target volume was used. For 1D SAGE, the $\mathrm{CF}$ was chosen from a $\mathrm{CF}$ versus measured $b$ curve; volume-dependent recovery-coefficient correction was invoked.

\section{RESULTS}

Convergence was investigated on the basis of total counts within the VoI for the target sphere. Twenty iterations was previously sufficient for 1D SAGE, and was also found to be enough with 3D OSEM for the experimental data acquired with either the UHE or HE (Fig. 1).

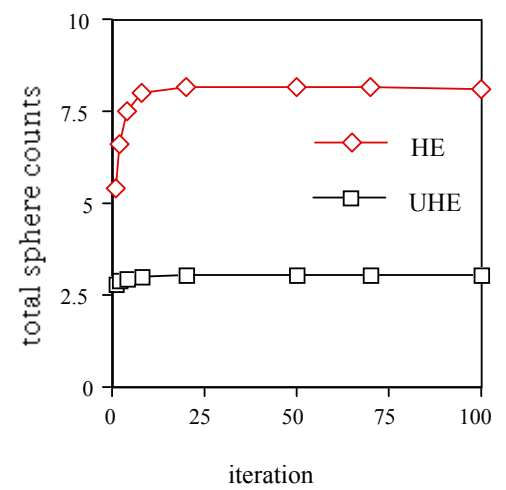

Fig. 1. Total-VoI-counts convergence for $200 \mathrm{~cm} 3$ sphere. comparison of UHE versus HE. Thicker (x2) UHE septa cause lower efficiency (less counts).

The convergence for smaller spheres with Monte-Carlo simulation of UHE data was found to vary with the situation. In Monte-Carlo simulations, one hundred iterations provided sphere-activity estimates independent of the sphere volume as shown in Fig. 2 and therefore was used for 3D OSEM reconstructions of all UHE data.

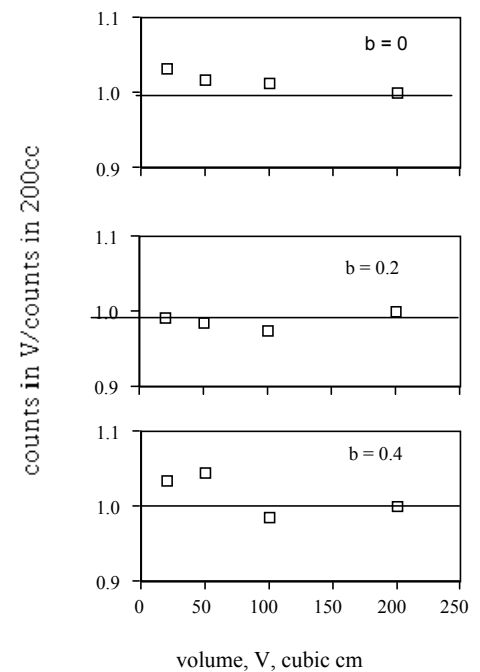

Fig. 2. Count ratio, small sphere over $200 \mathrm{cc}$ sphere, versus volume, V, of small sphere.

For the UHE collimator using 3D OSEM, CF varied linearly with b (Fig. 3). Units for CF are counts per (microcurie sec). The time in seconds is the total time of the acquisition. (This total time is the acquisition time for one stop times the number of stops. Moreover, it is further assumed that the data from all three camera heads contributes to the reconstruction.). With 1D SAGE, CF again exhibited a linear dependence on measured $b$ for each $R$, but the absolute value of the slope was much larger than with 3D OSEM (for $\mathrm{R}=23 \mathrm{~cm}$, the absolute value of the slope was 2.67 times as large ( 0.310 divided by 0.116)). A sample dependence on b is shown in Fig. 3. At 
$\mathrm{b}=0$, there are more counts with 3D OSEM due to superior resolution recovery (3D OSEM is inherently unregularized while 1D SAGE is regularized). With the largest background $(b=0.37)$, count spill in from the background with 1D SAGE has brought the sphere count up to equal that with 3D OSEM. Results were similar at four other values of the radius of rotation, $\mathrm{R}$.

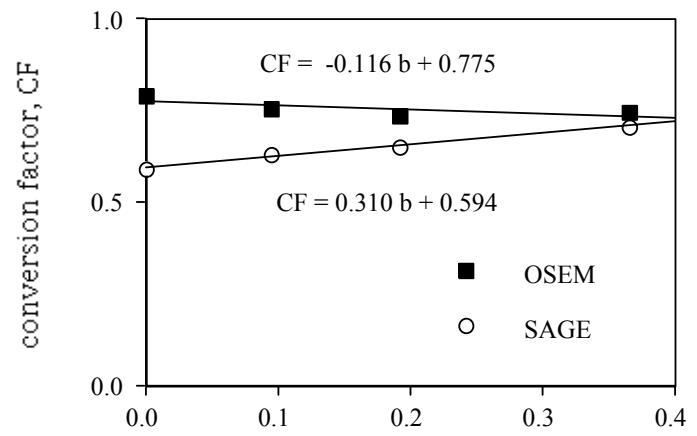

background-to-target activity-concentration ratio, $\mathrm{b}$

Fig. 3. $C F$ versus $b$ for the UHE collimator. $R=23 \mathrm{~cm}$.

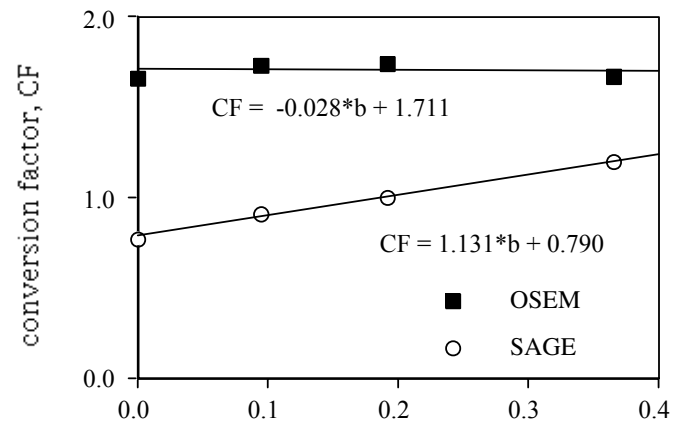

background-to-target activity-concentration ratio, $\mathrm{b}$

Fig. 4. $\mathrm{CF}$ versus $\mathrm{b}$ for the $\mathrm{HE}$ collimator. $\mathrm{R}=22 \mathrm{~cm}$.

The equivalent plot with the HE collimator is shown in Figure 4. Note that with 3D OSEM the slope with the HE is much smaller than with the UHE. Also, for the HE the absolute value of the slope with 3D OSEM is much less than with $1 \mathrm{D}$ SAGE (0.028 compared to 1.131 at this radius). At the other measured radius of rotation the slope over intercept ratio with OSEM for HE collimation is similar to that for UHE collimation using OSEM. The change with radius for HE collimation isn't understood.

The bias in the activity estimate for the lung sphere is given in Table 1 below:

TABle 1. Mean BIAS AND RELATIVE STANDARD DEVIATION OF THAT BIAS FOR HE AND UHE COLLIMATION AS A FUNCTION OF QUANTIFICATION METHOD.

\begin{tabular}{|c|c|c|c|c|}
\hline \multirow{2}{*}{$\begin{array}{c}\text { Quantification } \\
\text { method }\end{array}$} & \multicolumn{2}{|c|}{ UHE collimation } & \multicolumn{2}{c|}{ HE collimation } \\
\cline { 2 - 5 } & mean & $\begin{array}{c}\text { Relative } \\
\text { standard } \\
\text { deviation }\end{array}$ & mean & $\begin{array}{c}\text { Relative } \\
\text { standard } \\
\text { deviation }\end{array}$ \\
\hline 3D OSEM & $-7.4 \%$ & $5.9 \%$ & $-9.4 \%$ & $3.8 \%$ \\
\hline 1D SAGE & $-10.7 \%$ & $2.4 \%$ & $-4.3 \%$ & $7.4 \%$ \\
\hline
\end{tabular}

\section{DISCUSSION}

The UHE collimator and 3D OSEM reconstruction appear to provide high resolution as judged by the good activity recovery for small spheres. This resolution should lessen spillover from background activity. The negative (rather than 0 ) slope for the UHE CF versus b curve is apparently an anomaly of how $3 \mathrm{D}$ OSEM reconstructs different activity distributions. With HE collimation, zero background and 3D OSEM, the total counts for the large sphere compared to the UHE result are more ( a factor of $2.2,1.711 / 0.790)$ than would be expected if both had only geometrically-collimated counts (a factor of $1.6[6]$ ) because 3D OSEM recovers septal-penetration counts present with the HE but not with the UHE. Imperfect recovery of such counts to the background region may explain why the CF doesn't decrease as much with $b$ for HE collimation at $\mathrm{R}=22 \mathrm{~cm}$, compared to the "natural" decrease shown by the UHE collimation (that is, imperfectly-recovered septalpenetration counts from the increasing background increment the sphere total for the HE). Alternatively, the result may again be a characteristic of 3D OSEM reconstruction. In any case, quantification of focal total activity is better than $10 \%$ with both collimators and 3D OSEM. The bias is less with 3D OSEM than with 1D SAGE-based activity quantification for the UHE collimation but not for HE collimation. The noise, as measured by the relative standard deviation of the bias, is greater with 3D OSEM than with 1D SAGE-based activity quantification for the UHE collimation but not for HE collimation. The testing of more anthropomorphic-phantom geometries is needed to further compare activity quantification using UHE collimation versus that using HE collimation. So far, the results are similar.

\section{REFERENCES}

[1] Fessler J, Hero A. Space alternating generalized expectationmaximization algorithm. IEEE Trans Signal Processing. 42: 2664$2677,1994$.

[2] Fessler, JA and Hero, AO. Penalized maximum-likelihood image reconstruction using space-alternating generalized EM algorithms. IEEE Tr. Im. Proc., 10:1417-29,1995.

[3] Koral KF, Dewaraja Y, Li J, Lin Q, Regan DD, Zasadny KR, Rommelfanger SG, Francis IR, Kaminski MS, and Wahl RL. "Update on hybrid conjugate-view SPECT tumor dosimetry and response in 131Itositumomab therapy of previously untreated lymphoma patients," J Nucl Med, vol. 44, pp. 457-64, 2003.

[4] Hudson HM, Larkin RS. Accelerated Image-Reconstruction Using Ordered Subsets of Projection Data. IEEE Trans Med Imaging, 13:601$609,1994$.

[5] Zeng GL, Gullberg GT. Frequency domain implementation of the threedimensional geometric point response correction in SPECT imaging. IEEE Trans Nuc Sci. 39:1444-1453,1992.

[6] Dewaraja YK, Ljungberg M, Koral KF. Accuracy of I-131 quantification in radioimmunotherapy using SPECT imaging with an ultra-high-energy collimator: Monte Carlo study. J Nucl Med 2000; 41:1760-1767.

[7] Koral KF, Lin Q, Yendiki A, Akhtar A, Dewaraja YK, Fessler JA. Application of unregularized OSEM with 3-D detector response to 131I SPECT with ultra-high- and high-energy collimation. J Nucl Med 43(5):215P, 2002. 
[8] Koral KF, Lin Q, Akhtar A, Li J, Dewaraja YK, Fessler JA. Effect of 3$\mathrm{D}$ detector response on SPECT quantification of focal 131I activity. Conference Record of the 2001 IEEE Medical Imaging Conference, San Diego, Nov. 4-10.

[9] Koral KF, Yendiki A, Lin Q, Dewaraja YK, Fessler JA. Determining Total I-131 activity within a VoI using SPECT, a UHE collimator, and 3D-Detector-Response OSEM. Submitted to the IEEE Transactions of Nuclear Science, 2003.

[10] Ljungberg M, Strand S-E. A Monte Carlo program simulating scintillation camera imaging. Comp Meth and Progr in Biomed. 29:257272,1989 .

[11] de Vries DJ, Moore SC, Zimmerman RE, Mueller SP, Friedland B, Lanza RC. Development and validation of a Monte Carlo simulation of photon transport in an anger camera. IEEE Trans Med Imag. 9:4304,1990 . 